\title{
Quantum Games in Open Systems using Biophysic Hamiltonians
}

\author{
Jean Faber ${ }^{1}$, Renato Portugal ${ }^{1}$ and Luiz Pinguelli Rosa ${ }^{2}$ \\ ${ }^{1}$ National Laboratory of Scientific Computing - LNCC, \\ Av. Getlio Vargas 333, Quitandinha, 25651-075, Petrpolis, RJ, Brazil. \\ \{faber, portugal\}@lncc.br \\ ${ }^{2}$ Federal University of Rio de Janeiro, COPPE-UFRJ, RJ, Brazil, \\ lpr@adc.coppe.ufrj.br
}

11th August 2018

\begin{abstract}
We analyze the necessary physical conditions to model an open quantum system as a quantum game. By applying the formalism of Quantum Operations on a particular system, we use Kraus operators as quantum strategies. The physical interpretation is a conflict among different configurations of the environment. The resolution of the conflict displays regimes of minimum loss of information.
\end{abstract}

\section{Introduction}

Recently, many papers have extended the game theory concepts to the quantum world [1]-[7. By quantizing a game, it is possible to improve its efficiency and stability [4. However, by looking at the relationship between physical systems and the Theory of Games (TGs) we may ask: What are the conditions to model a physical system in terms of a game?

In order to address this question we will turn our attention to the theory of open quantum systems. Open systems allow us to use Kraus operators as quantum strategies, which can be obtained by the formalism of Quantum Operations 8 . Our goal is to model open quantum systems using Quantum Games. We apply these concepts to the quantum biological model proposed by Frhlich 9, 10.

In section 2 we review the main concepts of quantum games. We emphasize the use of the Kraus operators as quantum strategies. In section 3 we discuss a biophysic Hamiltonian of an open system and explicitly calculate the Kraus operators associated with the coupling term of the Hamiltonian.

In section 4 we propose a game that uses the Kraus operators as quantum strategies and a general payoff criterion for open quantum systems. In section 5 we analyze numerically the equilibrium points in the Quantum Phase Damping Channel game model. In section 6 we analyze the possible consequences of this new proposal. Finally, in section 7 we draw conclusions.

\section{Extending the Quantum Strategies}

In quantum games the strategies usually belong to the group $S U(2)$. However, to obtain relevant quantum effects in a game it is enough to consider a 2-parameter unitary matrix defined by [2, 4], 6]

$$
U(\theta, \varphi)=\left(\begin{array}{cc}
e^{i \varphi} \cos (\theta / 2) & \sin (\theta / 2) \\
-\sin (\theta / 2) & e^{-i \varphi} \cos (\theta / 2)
\end{array}\right)
$$


where $\theta \in[0, \pi]$ and $\varphi \in[0, \pi / 2]$. Following the protocol of Eisert, Wilkens, and Lewenstein (EWL) 2], a quantum game of two players, $A$ and $B$, can be completely described by the set $\Omega=\left\{\mathcal{H}, \mathcal{S}_{A}, \mathcal{S}_{B}, \rho_{i n}, \mathcal{F}_{A}, \mathcal{F}_{B}\right\}$, where $\mathcal{H}$ is the Hilbert space of the quantum system, $\rho_{\text {in }}$ is the density matrix which defines the initial configuration of the game and $\mathcal{F}_{A,(B)}$ is the evaluation function or payoff function of the player $A(B)$, defined as

$$
\mathcal{F}_{A, B}\left(\left\{\theta_{A}, \varphi_{A}\right\},\left\{\theta_{B}, \varphi_{B}\right\}\right)=\operatorname{tr}\left(P_{A, B} \rho_{\text {fin }}\right),
$$

where $P_{A, B}=\sum_{i j} w_{i j}^{A, B}|i j\rangle\langle i j|$ are the payoff operators and $\rho_{f i n}=\left|\psi_{f i n}^{A B}\right\rangle\left\langle\psi_{f i n}^{A B}\right|$, with

$$
\left|\psi_{f i n}^{A B}\right\rangle=J^{\dagger}(\chi)\left(U\left(\theta_{A}, \varphi_{A}\right) \otimes U\left(\theta_{B}, \varphi_{B}\right)\right) J(\chi)|00\rangle,
$$

and $J(\chi)$ is a nonlocal unitary transformation defined by $J(\chi)=\cos (\chi / 2) I \otimes I+\sin (\chi / 2) D \otimes$ $D$, with $\chi \in[0, \pi / 2]$ controlling the entanglement degree, $I=U(0,0)$ and $D=U(\pi, 0)$.

Nevertheless, according to [2] and 4] every quantum game can be described as a Completely Positive Trace-Preserving Map (CPTPM). This characterization allows an important generalization: We can use Kraus operators as quantum strategies. In this direction, by using the formalism of Quantum Operations (QOs) [8], which defines a CPTPM, we will be able to model an open quantum systems as a game. QOs may describe different types of noise that different kinds of environmental configurations introduce into a quantum system. The noises are described by the Kraus operators which act on the principal system.

In this way, the solutions of a game are the equilibrium and the dominant points of the strategies. There are two important concepts [2], 3], 11]:

(i)Dominant Strategy (DE). It is the strategy that has the best performance in a game from the viewpoint of one player. It doesn't necessarily give the best payoff if all players adopt the DE.

(ii) Nash Equilibrium (NE). It is the pair of strategies $\left(\stackrel{\star}{s}_{A}, \stackrel{\star}{s}_{B}\right)$ from which neither player can improve their payoff by an unilateral change in strategy. Mathematically, a pair $\left(\stackrel{\star}{s}_{A}, \stackrel{\star}{s}_{B}\right)$ is in NE if

$$
\begin{aligned}
& \mathcal{F}_{A}\left(\stackrel{\star}{s}, \stackrel{\star}{s_{B}}\right)-\mathcal{F}_{A}\left(s_{A}, \stackrel{\star}{s} B\right) \geq 0, \quad \forall s_{A}, \\
& \mathcal{F}_{B}\left(\stackrel{\star}{s}_{A}, \stackrel{\star}{s}_{B}\right)-\mathcal{F}_{B}\left(\stackrel{\star}{s}_{A}, s_{B}\right) \geq 0, \quad \forall s_{B} .
\end{aligned}
$$

\section{Quantum Strategies from Hamiltonians}

In the sixties, Frhlich [9] developed a theoretical model focusing on some of electrooscillatory physical properties of biomolecular systems. He showed that it is possible to describe relevant quantum effects in those biosystems by approximating a group of proteins and its environment by a group of coupled harmonic oscillators. The complete Frhlich Hamiltonian is described in [10]. To simplify the model we consider only the Hamiltonian that describes the interaction between the principal system and the thermal bath

$$
H_{T B}=\hbar \xi\left(a^{\dagger} a b^{\dagger}+a^{\dagger} a b\right)
$$

where $a^{\dagger}$ and $a$ are the creation and the annihilation operators of the principal system, respectively; $b^{\dagger}$ and $b$ are the creation and annihilation operators of the thermal bath, respectively. The Hamiltonian $H_{T B}$ describes a coupling of second order between the principal system and the environment (the thermal bath), through a real coupling constant $\xi$. Both the principal system and the environment are modeled as harmonic oscillators.

In order to construct a game from the Hamiltonian (5) we must find the superoperators associated with the coupling term $\xi$. Each set of superoperators corresponds to an environment noise inserted into the principal system. Therefore, we can ask: What is the best 
environmental configuration that generates the smallest loss of information in the principal system? In other words, what is the best frequence of noise insertion that provides the maximum storage of information in the principal system?

From the definition of QOs, we obtain the general expression for the superoperators 8

$$
\mathbf{S}_{k}=\sum_{m} \sum_{n}\left\langle\mathfrak{A}_{m} \mathfrak{B}_{k}\left|U_{T B}\right| \mathfrak{A}_{n} \mathfrak{B}_{0}\right\rangle\left|\mathfrak{A}_{m}\right\rangle\left\langle\mathfrak{A}_{n}\right|,
$$

where $\left\{\left|\mathfrak{B}_{i}\right\rangle\right\}$ and $\left\{\left|\mathfrak{A}_{i}\right\rangle\right\}$ are the states of the thermal bath and the principal system respectively. The states of the environment are taken in the thermodynamic equilibrium and the states of the principal system are taken in a general high level of energy. The evolution operator $U_{T B}$ obey the Schrdinger equation associated with $H_{T B}$. Using the fact that $\left[a_{i}^{\dagger}, b_{j}^{\dagger}\right]=0, \forall i, j$, the relation $U_{T B}\left|\mathfrak{A}_{0} \mathfrak{B}_{0}\right\rangle=\left|\mathfrak{A}_{0} \mathfrak{B}_{0}\right\rangle$ and the Baker-Campbell-Hausdorf relation 8 , we obtain

$$
\begin{aligned}
U_{T B}\left|\mathfrak{A}_{n} \mathfrak{B}_{0}\right\rangle & =\sum_{m=0}^{\infty} \frac{(-i n t \xi)^{m}}{(m !)(n !)^{-1 / 2}}\left(b+b^{\dagger}\right)^{m}\left|\mathfrak{A}_{n} \mathfrak{B}_{0}\right\rangle \\
& =\sum_{m}^{\infty} \sum_{j=0}^{N-1} \frac{(-i n t \xi)^{m}}{(m !)(n !)^{-1 / 2}} g(m, j)\left|\mathfrak{A}_{n} \mathfrak{B}_{j}\right\rangle,
\end{aligned}
$$

where $g(m, j)$ are defined by $\left(b+b^{\dagger}\right)^{m}|0\rangle=\sum_{j=0}^{N-1} g(m, j)|j\rangle$ and are derived from the recurrent expression

$$
\left(b+b^{\dagger}\right)^{m+1}|0\rangle=\left(b+b^{\dagger}\right)\left(\sum_{j=0}^{N-1} g(m, j)|j\rangle\right) .
$$

For a quantum game it is enough to consider a system of only two levels of energy. Substituting $U_{T B}$ into (6) we obtain the superoperators relative to the second order coupling between the principal system and the thermal bath. For two levels we have to analyze the convergence of the series with the coefficient $g(m, j)$. For all even $m g(m, 0)=1$, for all odd $m g(m, 0)=0$ and the opposite for $g(m, 1)$.

Therefore, from []ㅣㅁ, we obtain

$$
\begin{gathered}
\mathbf{S}_{0}(\gamma)=\left|\mathfrak{A}_{0}\right\rangle\left\langle\mathfrak{A}_{0}|+\cos (t \xi)| \mathfrak{A}_{1}\right\rangle\left\langle\mathfrak{A}_{1}\right|=\left(\begin{array}{cc}
1 & 0 \\
0 & \sqrt{1-\gamma}
\end{array}\right) \\
\mathbf{S}_{1}(\gamma)=\sin (t \xi)\left|\mathfrak{A}_{1}\right\rangle\left\langle\mathfrak{A}_{1}\right|=\left(\begin{array}{cc}
0 & 0 \\
0 & \sqrt{\gamma}
\end{array}\right)
\end{gathered}
$$

where $\gamma=\sin ^{2}(t \xi)$.

The superoperators (10) define a mapping known as quantum phase damping channel (QPDC). This channel plays an important role because it models the effect of decoherence. In this case $\gamma$ can be interpreted as the probability that a photon has been scattered from a quantum system $[8$.

The point now is to construct a reward criterion to measure the information in the principal system. Physically it means to calculate directly the entropy of the principal system after having interacted with the environment. However, we cannot say what environment configuration provides the optimal points of information storage. Using the TGs we will be able to analyze these optimal points by comparing two different environmental configurations. The principal advantage of this method is the direct association of a physical system with an information analysis. Besides, it provides a new interpretation of open quantum systems as a kind of game, showing a hidden optimization procedure in such systems. 


\section{A Payoff Based on the Information of the System}

In order to construct a payoff criterion, we calculate the information of the principal system after interaction with the environment (for each space, $A$ and $B$ ). To do this, we use the concept of information as described in Refs. 8] and [10. If the superoperators represent strategies, the rewards will be given according to the loss information of the principal system produced by each pair of strategy (from the players $A$ and $B$ simultaneously). If the player $A$ plays a strategy $s_{i}^{A} \in \mathcal{S}_{A}$ and the player $B$ plays a strategy $s_{k}^{B} \in \mathcal{S}_{B}$, the joint information is given by

$$
I\left(s_{i}^{A}, s_{k}^{B}\right)=-\log _{2}\left(p_{i k}^{A B}\right) \forall i, k,
$$

where $p_{i k}^{A B}$ is the joint probability associated with a quantum noise from the simultaneous occurrence of the pair $s_{i}^{A}$ e $s_{k}^{B}$. This probability measures the degree of dependence between the strategies of the players ${ }^{1}$. If the strategies of $A$ and $B$ are measurement operators, the joint probabilities are given by

$$
p_{i k}^{A B}=\operatorname{tr}\left(s_{i}^{A} \otimes s_{k}^{B}\left(\rho_{i n}^{\prime}\right) s_{i}^{A \dagger} \otimes s_{k}^{B \dagger}\right)
$$

where in the game context $\rho_{i n}^{\prime}=J(\chi) \rho_{\text {in }} J^{\dagger}(\chi)$ such that $\rho_{\text {in }}=\left|\psi_{i n}^{A B}\right\rangle\left\langle\psi_{i n}^{A B}|, \quad| \psi_{i n}^{A B}\right\rangle=$ $\left|\mathfrak{A}_{0}^{A} \mathfrak{A}_{0}^{B}\right\rangle \equiv|00\rangle$ and the set of strategies of $A$ and $B$ is constructed from the superoperators (10).

From the information theory, we know that events with small chance to happen have high information and vice versa. Therefore, by associating the joint information to each element of the payoff matrix, we obtain a good criterion to reward the "player's choice". Of course the environment does not choose its strategies. The actions are physically equivalent to a random insertion of noise into the principal system. Thus, in the computational basis we can define the payoff operators by

$$
P_{A, B}=\sum_{i k} w_{i k}^{A, B}|i k\rangle\left\langle i k\left|=\sum_{i, k=0}^{1} I\left(s_{i}^{A}, s_{k}^{B}\right)\right| i k\right\rangle\langle i k|,
$$

where $I\left(s_{i}^{A}, s_{k}^{B}\right)=I\left(s_{k}^{B}, s_{i}^{A}\right), \forall i \neq k$, producing a symmetric game.

This criterion introduces a natural cooperation between the players since the optimal points are reached by pairs of strategies. Besides, this payoff criterion can be seen as a kind of "information measurement" applied to open quantum systems since the payoff function corresponds to an average over the amount of information associated with the quantum noise probabilities. It expresses naturally the amount of information in the interface between the quantum and the classical worlds. Hence, we can apply this criterion in every quantum game that uses measurement operators as quantum strategies ${ }^{2}$.

\section{An Open System as a Quantum Game}

If we use the superoperators as quantum strategies the noise insertion can be seen as players applying their strategies. By considering two different environmental configurations, $A$ and $B$, we retrieve two players. That is, the noise insertion from each environment represents the strategic action of each player.

Since the superoperators (10) satisfy the condition $\sum_{k} \mathbf{S}_{k} \mathbf{S}_{k}^{\dagger}=\mathbf{1}$, we label the set of strategies of the players $A$ and $B$ by $\mathcal{S}_{A}=\left\{s_{0}^{A} \equiv \mathbf{S}_{0}\left(\gamma_{A}\right), s_{1}^{A} \equiv \mathbf{S}_{1}\left(\gamma_{A}\right)\right\}$ and $\mathcal{S}_{B}=\left\{s_{0}^{B} \equiv\right.$

\footnotetext{
${ }^{1}$ To avoid singularities, we limited the logarithm function by $\left|\lim _{\varepsilon \rightarrow 0}\left(-\log _{2}(\varepsilon)\right)\right| \leq \delta, \forall \delta \geq 0$. For numerical calculations we assume the minimum as $\varepsilon=10^{-10}$.

${ }^{2}$ For unitary operations an alternative criterion might be given by the Von Neumann Entropy 8 . The payoffs would be constructed from $w_{i k}^{A, B}=-\operatorname{tr}_{\mathrm{B}, \mathrm{A}}\left(\phi \log _{2} \phi\right)$, where $\phi=\left(U_{i}^{A} \otimes U_{k}^{B}\right) \rho\left(U_{i}^{A} \otimes U_{k}^{B}\right)^{\dagger}$.
} 
$\left.\mathbf{S}_{0}\left(\gamma_{B}\right), s_{1}^{B} \equiv \mathbf{S}_{1}\left(\gamma_{B}\right)\right\}$, respectively. In this way, from the definition of QOs and from the EWL protocol, the final state of the game is

$$
\rho_{\text {fin }}=J^{\dagger}(\chi) \sigma J(\chi)
$$

where

$$
\sigma=\sum_{i, k=0}^{1} s_{i}^{A} \otimes s_{k}^{B}\left(\rho_{i n}^{\prime}\right) s_{i}^{A \dagger} \otimes s_{k}^{B \dagger} .
$$

By taking the average of the operators (13) over $\rho_{\text {fin }}$ we calculate the payoff function of each player. The possible variations of the strategies are introduced into the quantum noise terms $\left(\gamma_{A}\right.$ and $\left.\gamma_{B}\right)$. This process shows the quantum nature of the game ${ }^{3}$.

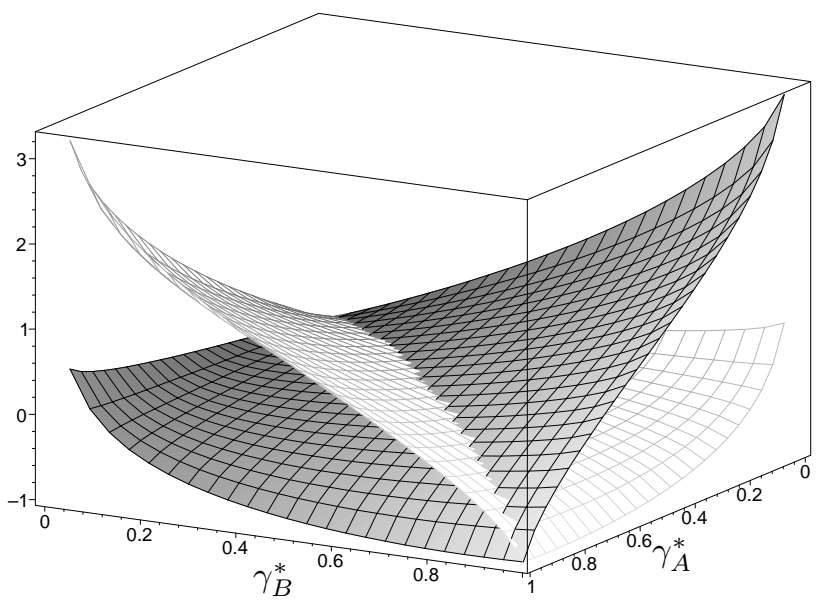

Figure 1: NE points for $\left(\gamma_{A}, \gamma_{B}\right)=(0.1,0.1)$ where $\left(\gamma_{A}^{\star}, \stackrel{\gamma}{\gamma}_{B}^{\star}\right)_{\mathrm{MAX}} \approx(0.4,0.4)$.

For a noise given by a QPDC, the channel $A$ plays against $B$ manipulating the states of the principal system through their set of strategies $\mathcal{S}_{A}$ and $\mathcal{S}_{B}$. In this way, we construct a game for the QPDC from $H_{T B}$. However, from the rules of the superoperators (10) and since $J(\chi)|00\rangle=\cos (\chi / 2)|00\rangle+i \sin (\chi / 2)|11\rangle$, the game model will not include the worst case in a QPDC that occurs when the state is $(|0\rangle+|1\rangle) \otimes(|0\rangle+|1\rangle) / 2$. To outline this problem, we apply the entanglement operator $J_{P D}(\chi) \equiv J(\chi)(H \otimes H)$ instead of $J(\chi)$ in EWL protocol. This new procedure does not modify the essential structure of a quantum game and does not decrease the entanglement degree ${ }^{4}$.

By applying (13) over the final state (14), and considering a general $\chi$, we obtain the

\footnotetext{
${ }^{3}$ In a open quantum system the operator $J^{\dagger}$ can be seen as a basis change of the payoff operators.

${ }^{4}$ Since $D=U(\pi, 0)=|0\rangle\langle 1|-| 1\rangle\langle 0|$, we have $J_{P D}(\chi)|00\rangle=(-1)^{\chi / 2 \pi}(|00\rangle+|11\rangle) / 2+(-1)^{-\chi / 2 \pi}(|01\rangle+$ $|10\rangle) / 2$. Besides, $\left[J, s_{i}^{x} \otimes s_{j}^{y}\right]=\left[J_{A D}, s_{i}^{x} \otimes s_{j}^{y}\right]=\left[J_{P D}, s_{i}^{x} \otimes s_{j}^{y}\right]=0, \forall i, j$ only when $s_{i}^{x}$ is equal to a generic classical strategy $U_{i}\left(\theta_{x}, 0\right)$.
} 
payoff function of the player $A$ :

$$
\begin{gathered}
\mathcal{F}_{A}\left(\gamma_{A}, \gamma_{B}\right)=I\left(s_{0}^{A}, s_{0}^{B}\right)\left(\sqrt{1-\gamma_{A}}+\sqrt{1-\gamma_{B}}+1+\sqrt{1-\gamma_{A}} \sqrt{1-\gamma_{B}}\right) / 4 \\
+I\left(s_{0}^{A}, s_{1}^{B}\right)\left[\sqrt{1-\gamma_{A}}-\sqrt{1-\gamma_{B}}+1-\sqrt{1-\gamma_{A}} \sqrt{1-\gamma_{B}}\right. \\
\left.+8\left(\sqrt{1-\gamma_{A}}-\sqrt{1-\gamma_{B}}\right) \cos (\chi / 2)^{2}+8\left(\sqrt{1-\gamma_{B}}-\sqrt{1-\gamma_{A}}\right) \cos (\chi / 2)^{4}\right] / 4 \\
+I\left(s_{1}^{A}, s_{0}^{B}\right)\left[\sqrt{1-\gamma_{B}}-\sqrt{1-\gamma_{A}}+1-\sqrt{1-\gamma_{A}} \sqrt{1-\gamma_{B}}\right. \\
\left.+8\left(\sqrt{1-\gamma_{A}}-\sqrt{1-\gamma_{B}}\right) \cos (\chi / 2)^{2}+8\left(\sqrt{1-\gamma_{B}}-\sqrt{1-\gamma_{A}}\right) \cos (\chi / 2)^{4}\right] / 4 \\
+I\left(s_{1}^{A}, s_{1}^{B}\right)\left(\sqrt{1-\gamma_{A}} \sqrt{1-\gamma_{B}}-\sqrt{1-\gamma_{B}}-\sqrt{1-\gamma_{A}}+1\right) / 4,
\end{gathered}
$$

where the joint information of each pair of strategies are calculated from (11) and (12). Similarly, the same calculation can be performed for the player $B$.

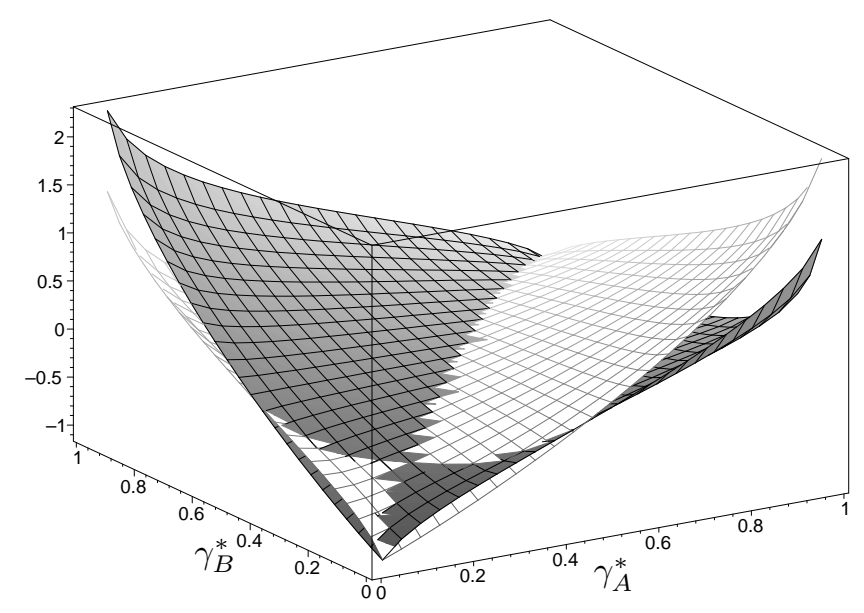

Figure 2: NE points for $\left(\gamma_{A}, \gamma_{B}\right)=(0.5,0.5)$ where $\left(\gamma_{A}^{\star}, \stackrel{\gamma}{*}_{B}^{\star}\right)_{\mathrm{MAX}} \approx(0.8,0.8)$.

To calculate the points of equilibrium we perform a numerical analysis by using the Nash equilibrium concept (4a) and (4b). By considering the initial state with a maximal entanglement $J_{P D}(\pi / 2)|00\rangle$ we calculate the pairs $\left(\stackrel{(}{\gamma}_{A},,_{B}^{\star}\right)$ for four values of $\left(\gamma_{A}, \gamma_{B}\right)$ that generate the NE points. Figures 1 to 4 show the points where the vertical axis of the colorless surfaces corresponds to the best equilibrium points (4a) of the player $A$; and the vertical axis of the colored surfaces corresponds to the best equilibrium points (4b) of the player $B$. The points $\left(\stackrel{\star}{\gamma}_{A},{\stackrel{\gamma}{\gamma_{B}}}^{\star}\right)$ in the intersection of the surfaces that have positive values in the vertical axis are Nash equilibrium points. (4b).

The colorless surface represents equation (4a) and the colored surface represents Equation

The graphics show the correspondence between the equilibrium points of the game and the physical stability of the system. By physical stability we mean a larger time of coherence. But, a larger time of coherence is related with a smallest probability of noise which is associated with the smallest values of the parameters of the strategies $\stackrel{\star}{\gamma}_{A, B} \sim 0$. It happens 


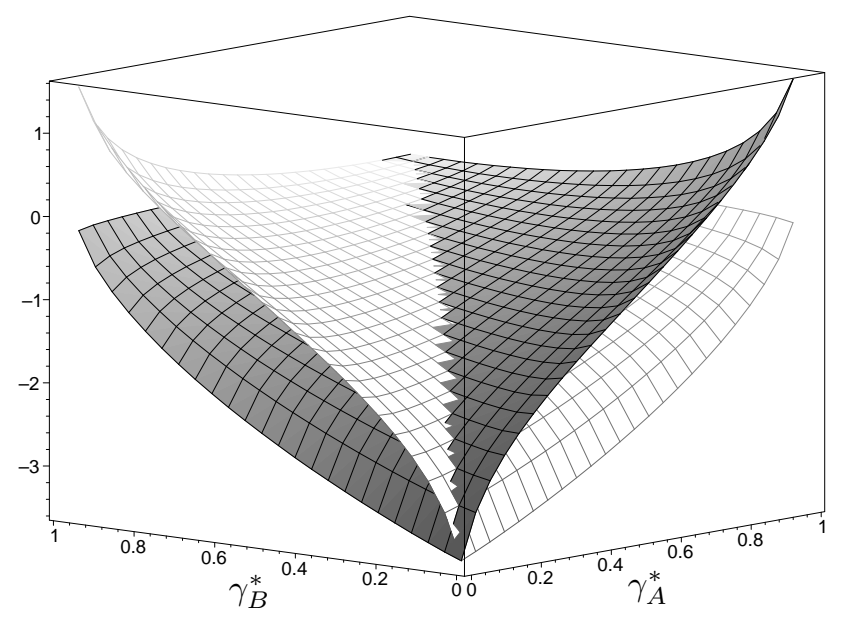

Figure 3: NE points for $\left(\gamma_{A}, \gamma_{B}\right)=(1,1)$ where $\left(\gamma_{A}^{\star}, \gamma_{B}^{\star}\right)_{\text {MAX }} \approx(1,1)$.

because the equilibrium points are directly associated with the optimal points of information storage in the principal system.

For small values of noise, $\left(\gamma_{A}, \gamma_{B}\right)=(0.1,0.1)$, the game shows NE points $\left(\gamma_{A}^{\star}, \stackrel{\gamma}{B}^{\star}\right)$ near to zero (Figure 1). For high values of noise, $\left(\gamma_{A}, \gamma_{B}\right)=(1,1)$, the game shows NE points near to one (Figure 3). An interesting result is achieved for $\left(\gamma_{A}, \gamma_{B}\right)=(0.5,0.5)$. The surfaces that characterize the equilibrium points are very close (Figure 21). And a natural asymmetry of dominance of game arise for different noise distribution, $\gamma_{A} \neq \gamma_{B}$, $\left(\gamma_{A}, \gamma_{B}\right)=(1,0.5)$, without eliminating the NE points (Figure 4). Besides, it is also possible to show the existence of NE points for $\left(\gamma_{A}, \gamma_{B}\right)=(0.1,1) ;\left(\gamma_{A}, \gamma_{B}\right)=(1,0.1)$ and for $\left(\gamma_{A}, \gamma_{B}\right)=(0.5,0.1) ;\left(\gamma_{A}, \gamma_{B}\right)=(0.1,0.5)$.

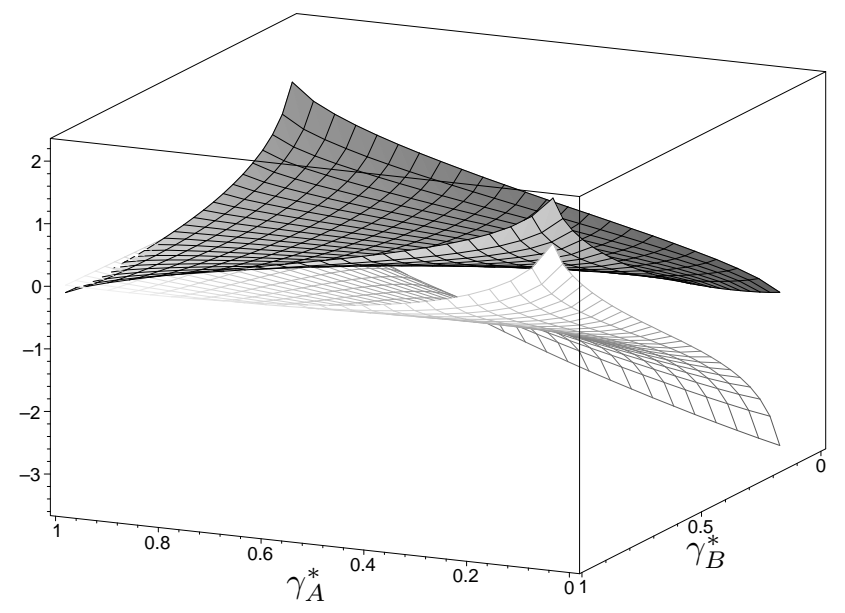

Figure 4: NE points for $\left(\gamma_{A}, \gamma_{B}\right)=(1,0.5)$ where $\left(\gamma_{A}^{\star}, \gamma_{B}^{\star}\right)_{\mathrm{MAX}} \approx(1,0.8)$.

The main conclusion is that even under intense noise the system may present physical 


\section{Discussion}

We discussed in section 3 that the Frhlich model describes the physical dynamics of a coupled biomolecule net. Inspired in this dynamics, the Hamiltonian of Frhlich has been used to model the physical relation of the neuronal microtubules [12. Several models claim that the microtubules process quantum information in a sub-neuronal level. It would help to produce certain mental functions such as the human consciousness [13] 15.

It is our belief that many physical systems can be solved by applying TGs to open quantum systems. Using the payoff criterion described in this paper, we have linked the information storage capacity to the physical stability points. Therefore, by using the game models, we think it is possible a biophysical analysis that shows the values of the relevant variables, such as the frequency and temperature, in the equilibrium points. These models are able to show whether there are or not equilibrium points close to the physiological temperature and close to the protein frequencies. This technique plays a fundamental role in describing any information processing in biophysical systems.

All these issues deserve a detailed description. However, we consider the TGs a very important tool to model, to calculate and to interpret any quantum information processing in open systems.

\section{Conclusion}

In this paper we show how to construct a quantum game from biophysical Hamiltonians. By using Kraus operators as quantum strategies, we provide a possible game interpretation of open quantum systems. In order to calculate the equilibrium points in the Frhlich quantum game we propose a new criterion for the payoff matrix focusing on the information associated with the principal system. By means of this criterion, the quantum game model can be interpreted as a game between two different environmental configurations which seek to minimize the noise insertion into the principal system. However, to improve the analysis of the equilibrium points, we have to incorporate some physical conditions. To analyze all points in the QPDC game we used the entanglement operator $J_{P D}(\chi)=J(\chi)(H \otimes H)$ instead of $J(\chi)$. Nevertheless, these extensions do not modify the essence of a quantum game. The model shows physical consistence and presents many points of stability, even under intense noises $\left(\gamma_{A, B} \sim 1\right)$.

Using this model the equilibrium of the game corresponds to the points of Nash equilibrium which determine the maximum regimes of information storage of the principal system. Therefore, the description of a physical system as a quantum game gives us not only a new interpretation but also a methodological alternative for evaluating the information storage points in open systems.

\section{References}

[1] D. A. Meyer, Phys. Rev. Lett. 82 (1999) 1052.

[2] J. Eisert, M. Wilkens, M. Lewenstein, Phys. Rev. Lett. 83 (1999) 3077.

[3] L. Marinatto, T. Weber, Phys. Lett. A 272 (2000) 291.

[4] S. C. Benjamim, P. M. Hayden, Phys. Rev. A 64 (2001) 030301. 
[5] J. Du, H. Li, X. Xu, M. Shi, J. Wu, X. Zhou, R. Han, J. Phys. A 36 (2003) 6551 .

[6] A. P. Flitney, D. Abbott, Fluct. Noise Lett. 4 (2002) R175.

[7] L. K. Chen, Huiling Ang, D. Kiang, L. C. Kwek, C. F. Lo, Phys. Lett. A 316 (2003) 317.

[8] M. A. Nielsen, I. L. Chuang, Quantum Computing and Quantum Information, Univ. Press, Cambridge (2000).

[9] H. Frhlich, Int. J. Quantum Chem. 2 (1968) 641.

[10] J. Pokorn, T-M. Wu, Biophysical Aspects of Coherence and Biological Order, Springer (1998).

[11] J. Hofbauer, K. Sigmund, Evolutionary Games and Population Dynamics, Univ. Press, Cambridge (1998).

[12] J. A. Tuszyński, J. A. Brown, P. Hawrylak, The Royal Society 356 (1998) 1897.

[13] J. Faber, R. Portugal, L. P. Rosa, BioSystems 83 (2006) 1.

[14] J. Faber, L. P. Rosa, Phys. Rev. E 70 (2004) 031902.

[15] S. Hagan, S. R. Hameroff, J. A Tuszynski, Phys. Rev. E 65 (2002) 061901. 